\title{
Fast Neutron Imaging with Semiconductor Nanocrystal Scintillators
}

Kyle M. McCall, Kostiantyn Sakhatskyi, Eberhard Lehmann, Bernhard Walfort, Adrian S. Losko, Federico Montanarella, Maryna I. Bodnarchuk, Franziska Krieg, Yusuf Kelestemur, David Mannes, Yevhen Shynkarenko, Sergii Yakunin, and Maksym V. Kovalenko*

Cite This: ACS Nano 2020, 14, 14686-14697

Read Online

ACCESS | Lلll Metrics \& More | 回 Article Recommendations

S1 Supporting Information

ABSTRACT: Fast neutrons offer high penetration capabilities for both light and dense materials due to their comparatively low interaction cross sections, making them ideal for the imaging of large-scale objects such as large fossils or as-built plane turbines, for which $\mathrm{X}$-rays or thermal neutrons do not provide sufficient penetration. However, inefficient fast neutron detection limits widespread application of this technique. Traditional phosphors such as $\mathrm{ZnS}$ : $\mathrm{Cu}$ embedded in plastics are utilized as scintillators in recoil proton detectors for fast neutron imaging. However, these scintillation plates exhibit significant light scattering due

Fast neutron imaging with colloidal NCs

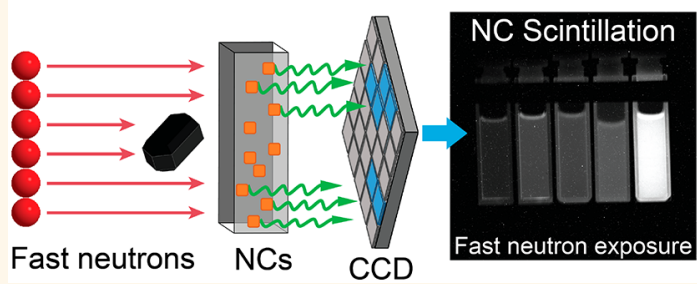
to the plastic-phosphor interface along with long-lived afterglow (on the order of minutes), and therefore alternative solutions are needed to increase the availability of this technique. Here, we utilize colloidal nanocrystals (NCs) in hydrogen-dense solvents for fast neutron imaging through the detection of recoil protons generated by neutron scattering, demonstrating the efficacy of nanomaterials as scintillators in this detection scheme. The light yield, spatial resolution, and neutron-vs-gamma sensitivity of several chalcogenide (CdSe and $\mathrm{CuInS}_{2}$ )-based and perovskite halide-based NCs are determined, with only a short-lived afterglow (below the order of seconds) observed for all of these NCs. FAPbBr 3 NCs exhibit the brightest total light output at $19.3 \%$ of the commercial $\mathrm{ZnS}: \mathrm{Cu}(\mathrm{PP})$ standard, while $\mathrm{CsPbBrCl}$ :Mn NCs offer the best spatial resolution at $\sim 2.6 \mathrm{~mm}$. Colloidal NCs showed significantly lower gamma sensitivity than $\mathrm{ZnS}: \mathrm{Cu}$; for example, $79 \%$ of the $\mathrm{FAPbBr}_{3}$ light yield results from neutron-induced radioluminescence and hence the neutron-specific light yield of $\mathrm{FAPbBr}_{3}$ is $30.4 \%$ of that of $\mathrm{ZnS}: \mathrm{Cu}(\mathrm{PP})$. Concentration and thickness-dependent measurements highlight the importance of increasing concentrations and reducing self-absorption, yielding design principles to optimize and foster an era of NC-based scintillators for fast neutron imaging.

KEYWORDS: nanocrystal scintillator, fast neutron detection, colloidal nanocrystal, halide perovskite, chalcogenide nanocrystal

S ince the discovery of X-rays by Roentgen in 1895, radiographic imaging has developed into a powerful nondestructive technique that has reshaped fields such as medical diagnostics, cargo and luggage security, and industrial product inspection. ${ }^{1-4}$ These successes have been driven by the variety of available radiation sources from X-rays and $\gamma$-rays to neutrons, as the materials' interaction cross sections depend on both the type and energy of the radiation. For example, the cross sections of X-rays drastically increase with increasing atomic number $\mathrm{Z}$ (Figure 1a), ${ }^{5}$ so while photons with X-ray energies are suitable for medical imaging, higher-energy $\gamma$-rays are more suitable for industrial applications. In contrast, the capture of neutrons has a complex dependence on the nuclear structure, with thermal neutrons $(\sim 25 \mathrm{meV}$ kinetic energy) providing good penetration for high-Z materials while remaining especially interactive with hydrogen-dense organic materials such as oil, water, or archeological specimens (Figure 1a). ${ }^{6}$ It should be noted that the values in Figure 1a are microscopic cross sections of individual nuclei in units of barns $\left(10^{-24} \mathrm{~cm}^{2}\right)$, the macroscopic cross sections $\left(\mathrm{cm}^{-1}\right)$ are obtained by multiplying by the number of nuclei per volume, known as the nuclear density. On the basis of the detection capability of low- $\mathrm{Z}$ materials in the presence of high- $Z$ materials, thermal neutron imaging has thus become an important nondestructive testing technique in aerospace, research, and defense applications. $^{3,7-10}$

Received: July 30, 2020

Accepted: September 8, 2020

Published: September 8, 2020 
a) Interaction Cross Section (barns)

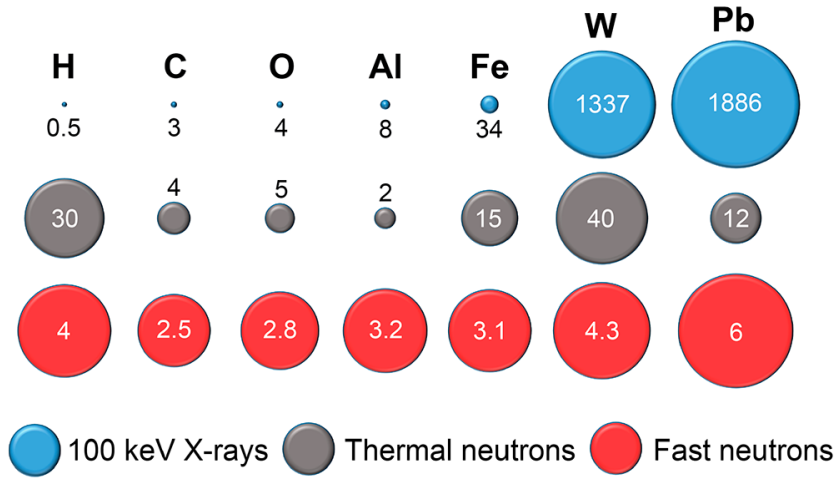

b) $10^{6}$

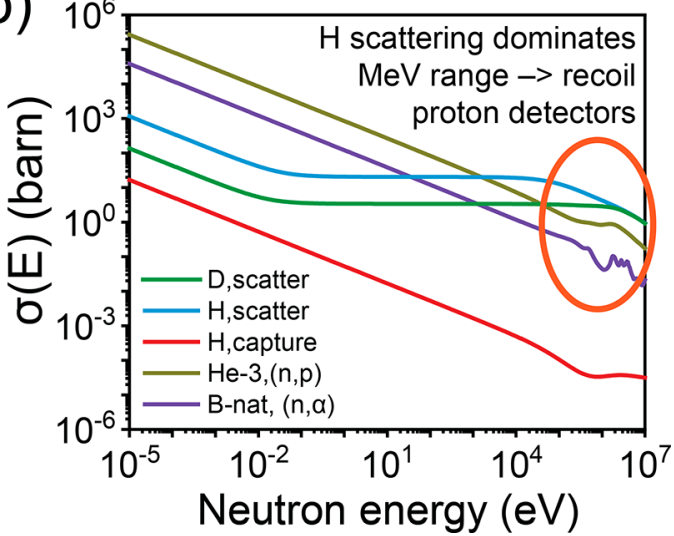

Figure 1. (a) Nuclei cross section comparison (proportional to the circle area, relative units for each radiation) of various chemical elements for $100 \mathrm{keV} \mathrm{X}$-rays, ${ }^{5}$ thermal neutrons $\left(25 \mathrm{meV}\right.$ ), ${ }^{6}$ and fast fission neutrons (average energy $\left.1.35 \mathrm{MeV}\right) .^{11}$ (b) $\mathrm{Nuclei} \mathrm{cross}$ sections of important neutron detection elements vs neutron energy, ${ }^{6}$ highlighting the scattering efficiency of hydrogen for fast neutrons.

Fast neutrons (kinetic energy higher than $1 \mathrm{MeV}$ ) offer higher penetration depths, with low total cross sections that do not depend so significantly on the material composition (Figure $1 \mathrm{a}) ;^{11}$ instead, contrast is typically provided by differences in nuclear density. Fast neutron imaging is a complementary technique that enables investigation of largerscale samples of dense, mixed-Z compositions, for which imaging with $\mathrm{X}$-rays, $\gamma$-rays, or even thermal neutrons would not provide sufficient penetration. ${ }^{12}$ Fast neutron imaging widens the scope of potential imaging applications to include cargo contraband detection or imaging of large archeological specimens or irradiated nuclear fuel. ${ }^{13-16}$ However, this technique has thus far been underutilized due to the limited number of fast neutron sources, and more critically the significant challenge of efficiently detecting fast neutrons.

The high penetration depth of the fast neutrons is advantageous for imaging large objects, but also limits the detection efficiency. For large detectors, that do not require spatial information, fast neutrons can be moderated to thermal energies for easier detection. ${ }^{17}$ However, this approach comes at the expense of poor spatial resolution due to the inherent principle of down-scattering, and therefore moderated fast neutrons are very limited for imaging applications. Traditional thermal neutron detectors have been based on particular isotopes with large absorption (capture) cross sections at low energy (mainly $\left.{ }^{3} \mathrm{He},{ }^{10} \mathrm{~B},{ }^{6} \mathrm{Li}\right)^{7,18}$ that efficiently produce more easily detected secondary particles. These cross sections are, however, three orders of magnitude lower at fast neutron energies (Figure 1b). ${ }^{6}$ Instead, the primary method for fast neutron detection is based on elastic scattering of neutrons by nuclei, generating recoil nuclei with very small penetration depths (up to tens of micrometers ${ }^{19}$ ) in the detector material. The kinetic energy of these recoil nuclei is then deposited as ionized charge carriers in the detector material, which can excite a scintillator to emit visible range photons detectable by conventional imaging devices such as a CCD camera. ${ }^{12}$ The energy transfer efficiency for elastic collisions is highest for two bodies of equal mass, thus the proton in a hydrogen atom is the best nucleus for fast neutron scattering. In conjunction with the relatively high interaction cross section of a proton with fast neutrons (Figure 1b), hydrogen-rich materials have been widely used in fast neutron detectors.

Consequently, there are two common types of fast neutron imaging detectors: an integrated liquid or plastic scintillator which provides both high proton density and light emission, ${ }^{7,20-23}$ or a two-part detector consisting of a hydrogen-dense matrix (often a plastic or polymer such as polypropylene (PP) or high-density polyethylene) and a scintillator material such as $\mathrm{ZnS}: \mathrm{Ag}, \mathrm{ZnS}: \mathrm{Cu}$, or $\mathrm{Gd}_{2} \mathrm{O}_{2} \mathrm{~S}: \mathrm{Tb}^{24-26}$ The latter type has shown the most success to date, ${ }^{12,27}$ with screens consisting of $\mathrm{ZnS}: \mathrm{Cu}$ in a PP matrix, denoted hereafter as $\mathrm{ZnS}: \mathrm{Cu}(\mathrm{PP})$, showing a good combination of light output and spatial resolution that has led to their commercialization by RC Tritec AG. ${ }^{12,28}$ However, long exposure times are still required to obtain high-quality images even under high fast neutron fluxes, while spatial resolution decreases substantially with thickness of the scintillator plate, ${ }^{28}$ likely due to light scattering at the phosphor-plastic interface, as the typical size of phosphor inclusions are larger than the emission wavelength. Another fundamental drawback is the afterglow of the $\mathrm{ZnS}: \mathrm{Cu}$ phosphor, which exhibits a several minute decay under fast neutron beam exposure, ${ }^{28}$ problematic for the short repeated exposures required for computed tomography and precluding the use of such screens in pulsed neutron experiments with high repetition rates. These limitations motivate the investigation of alternative concepts and materials that could enable the widespread application of fast neutron imaging detectors.

Quite separately, great strides have been made in the past several decades in semiconducting nanocrystals (NCs) for various applications such as LEDs, lasers, and single-photon sources. $^{29,30}$ The emergence of the CdQ ( $Q$ is a chalcogenide) nanocrystals in the $1990 \mathrm{~s}^{31}$ sparked immense interest in colloidal NCs that has come to encompass a wide variety of compounds from the traditional II-VI and III-V semiconductors to the ascendant halide perovskites. ${ }^{29,30,32}$ The success of semiconductor NCs has been driven by the effects of quantum confinement, leading to efficient emission and tunable optical and electronic properties that change with NC size. ${ }^{29,30,33,34}$ Further knobs for tuning properties have been discovered by the engineering of nanostructured materials with different morphologies such as nanoplatelets or core/shell structures. ${ }^{35-40}$

Despite the high photoluminescence quantum yields (PLQY) of NC emitters, only limited investigations have pursued radiation detection utilizing nanocrystalline materials as scintillators. ${ }^{41-44}$ The light yield achieved in NC scintillators has not yet matched the level expected from their outstanding 
PLQYs, ${ }^{41}$ possibly due to the increase in Auger recombination efficiency observed in CdSe NCs under $20 \mathrm{keV}$ excitation, limiting the radiative efficiency. ${ }^{45} \mathrm{~A}$ further obstacle for the use of NCs in scintillator detectors is the small loading (active volume) provided by such particles, requiring an active absorber matrix (e.g., a $\mathrm{Cs}_{4} \mathrm{PbBr}_{6}$ host for $\mathrm{CsPbBr}_{3} \mathrm{NCs}^{46}$ ) to provide the necessary stopping power to penetrative radiation such as X-rays and thermal neutrons. Recent reports of scintillation in perovskite NCs have utilized low-energy Xrays $(\sim 10 \mathrm{keV})$ to achieve radioluminescence and $\mathrm{X}$-ray imaging; ${ }^{47-49}$ however, efficient high-energy photon detection has to date been limited to single-crystal perovskite semiconductor detectors. ${ }^{50}$

However, as efficient emitters NCs hold great promise as the scintillator in an indirect detection scheme, such as the recoil proton detectors currently utilized for fast neutron imaging. An important advantage of NCs over bulk material is a high recombination rate due to quantum confinement for much faster luminescence decay times, as shown with X-ray excitation. ${ }^{51}$ Colloidal NCs are also small enough to lack the scattering that plagues phosphor-plastic detectors and can be dispersed in shape-engineered vessels, while regeneration of detectors through solution exchange would be beneficial for high radiation flux operation.

In this work, we design such a recoil proton detector through the use of colloidal NCs in solvents with high hydrogen-density and demonstrate fast neutron imaging with the use of nanoscale emitters. A variety of $\mathrm{NC}$ systems from the traditional CdSe-based materials to the $\mathrm{APbX}_{3}[\mathrm{~A}=$ formamidinium (FA), Cs; $\mathrm{X}=\mathrm{Br}, \mathrm{Cl}$ ] halide perovskites are screened for their light yield and spatial resolution under fast neutron irradiation, with $\mathrm{FAPbBr}_{3}$ providing the highest light yield, while $\mathrm{CsPbBrCl} 2 \mathrm{Mn} \mathrm{NCs}$ offer the best spatial resolution. Concentration and thickness-dependent measurements reveal that self-absorption and low concentrations are the primary limiting factors in these scintillators, providing design principles to foster the development of next-generation fast neutron detectors based on colloidal semiconductor NCs.

\section{RESULTS AND DISCUSSION}

Colloidal NCs for Recoil Proton Detection and Fast Neutron Imaging: Principles and Schematic. Fast neutron imaging through recoil proton detection with colloidal NCs is depicted in Figure 2a, and involves three steps. First, an incident fast neutron scatters off the $\mathrm{H}$-rich solvent or $\mathrm{H}$ containing NCs, generating a recoil proton. This high-energy recoil proton then deposits energy into the solvent or NC, creating a charge cloud of electrons and holes. These ionized charge carriers then excite the NC, which then undergo recombination and emit light to be detected by the CCD camera.

The overall detection efficiency is theoretically limited by the product of the individual efficiencies of these three processes, namely the fraction of fast neutrons scattered by the solvent, the charge conversion efficiency (CCE) that describes how the ionized charges excite the NC, and the quantum efficiency of the emission. The rate of neutron scattering is optimized by using solvents with high-hydrogen-density, while the efficiency of the NC emission is given by the PLQY. The CCE is not presently known for NCs in this indirect scheme, though recent work in electro- and cathodoluminescent NCs probes similar charge injection processes. ${ }^{45,52}$
Thus, the design of colloidal NC systems for fast neutron imaging relied chiefly on two parameters: solvents with high proton density and NCs with high PLQYs. The hydrogen density of the chosen solvents is compared with those of several plastics and polymers (Table S1). Despite being in the liquid state, the hydrogen density of the solvents is not far from their chemically similar polymer counterparts (i.e., aromatic toluene and polystyrene exhibit similar hydrogen densities, while those of cyclohexane and polypropylene are within 20\%). Hence, the proton-saturated alkane solvents scatter 22.8$24.1 \%$ of fast neutrons in a $1 \mathrm{~cm}$ sample, and were the preferred solvents where possible.

The chosen NC materials were selected from the chalcogenide and halide emitters which have proven to have excellent PLQYs. CdSe-based NCs, in particular, have the benefit of tunable morphology and core/shell structures due to their robust surface chemistry, ${ }^{35,39}$ while the chemically soft halide perovskites benefit from defect tolerance for high PLQYs. ${ }^{32}$ Hence, we chose seven systems for this investigation: (1) CdSe/CdS NCs, (2) CdSe/CdS/ZnS NCs, and (3) $\mathrm{CdSe} / \mathrm{CdZnS}$ nanoplatelets (NPLs) to compare the effects of the surface structure and morphology, (4) $\mathrm{CuInS}_{2} / \mathrm{ZnSe} /$ $\mathrm{ZnS}$ NCs for a higher Stokes-shift chalcogenide system, (5) $\mathrm{FAPbBr}_{3}$ and (6) $\mathrm{CsPbr}_{3} \mathrm{NCs}$ to compare the effect of the Asite cation, and finally (7) the doped perovskite $\mathrm{CsPbBrCl}_{2}: \mathrm{Mn}$ with high Stokes shift $\mathrm{Mn}^{2+}$ emission. Preparation of these NCs is described in the Supporting Information, and the solvents and photoluminescence characteristics of these NC materials are summarized in Table S2. Note that $\mathrm{FAPbBr}_{3} \mathrm{NCs}$ prepared at similar concentration in cyclohexane began to precipitate after a week in solution, requiring the use of the less hydrogen-dense toluene for these measurements.

Fast neutron imaging measurements were conducted at the NECTAR (NEutron Computed Tomography And Radiography) beamline of the FRM-II reactor in Garching, Germany; $^{53}$ a schematic of the instrument is found in Figure $2 b$. Briefly, a nuclear reactor core (1) generates neutrons which then excite a uranium silicide fast fission converter plate (2) to generate a fast neutron beam with an average neutron energy of 1.8 MeV. A series of filters and shutters (3) control and modulate the beam; in this work none of the optional filters were applied to the fast neutron beam to obtain maximum flux. A sample stage (4) allows for translation and rotation of objects to be imaged. Finally, the beam passes through a thin aluminum plate to the scintillator enclosure (5) consisting of a light-tight box and a CCD camera system which collects the incident light from the excited NC solutions in cuvettes. The cuvettes are mounted directly on the aluminum plate in the fast neutron beam path, while mirrors and lenses permit the camera to be out of the beam path for reduced background signal. A macroscopic view of this detection scheme is found in Figure $2 c$, along with the resulting fast neutron radiograph image obtained by the same NC sample cuvettes from the image in Figure 2b.

Light Yield Measurements and Afterglow Test. A series of comparison radiographs were measured under fast neutron exposure for the colloidal NC solutions (e.g., Figure $3 a)$ to compare the light yield of each scintillator material, measured by the average response of the CCD camera response in the central area of the liquid in the cuvettes. Note that the PL peak energies of these NCs (Table S2) all fall within the range of $90+\%$ quantum efficiency of the CCD camera used here. ${ }^{54}$ Enhanced light output can be observed at 
1) Elastic neutron scattering

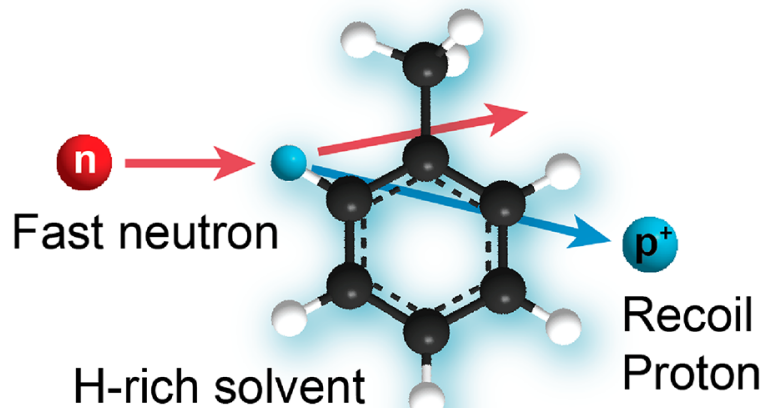

2) $p^{+}$ionization

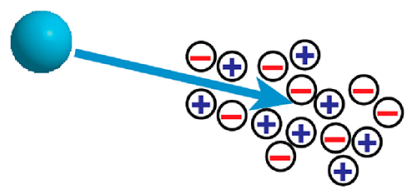

$\mathrm{e}^{-}-\mathrm{h}^{+}$pairs generated in or near NC
3) NC emission

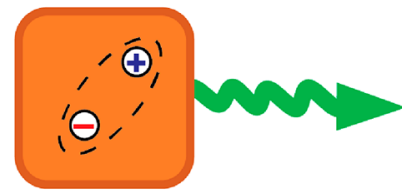

Recombination

$\rightarrow$ Emission

b)

Fast neutron imaging at NECTAR beamline

(1)

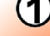

(1)

(2)

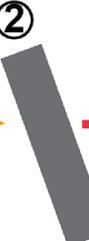

(1) Reactor core

(2) Fast fission converter plate

(3) Beam shutters

(4) Tomography sample stage

(5) Scintillator enclosure with CCD camera
(4)

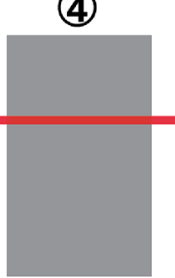

(5)
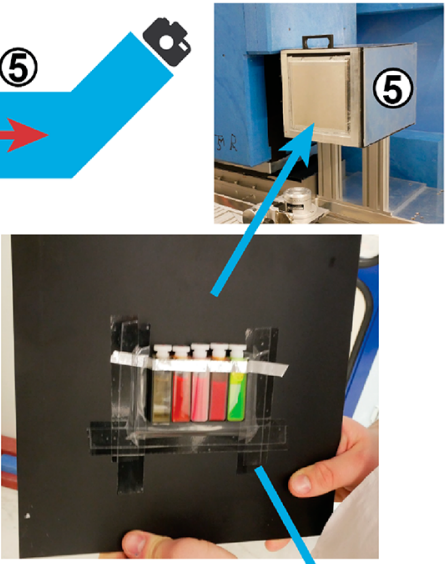

c)

Colloidal NC scintillators for fast neutron imaging

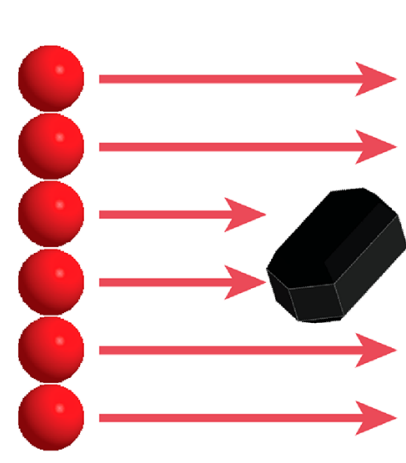

Fast neutron beam

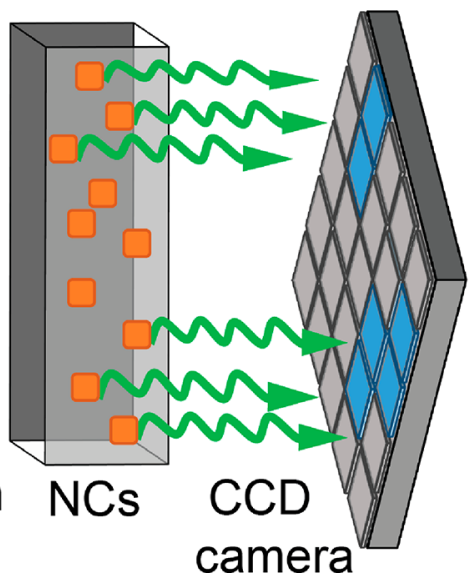

Colloidal NC Scintillation

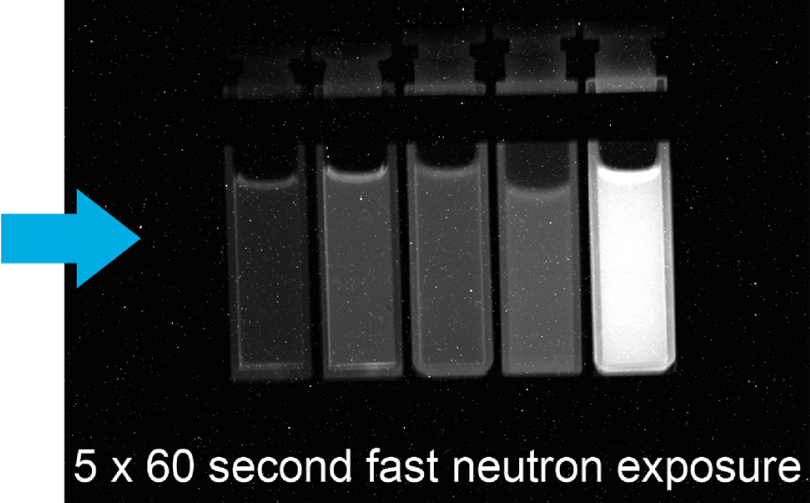

Figure 2. (a) Principles of fast neutron detection via recoil proton detection, depicting (1) elastic scattering of the fast neutron off an $\mathrm{H}$ atom of the solvent to generate a recoil proton, (2) the generation of a charge carrier cloud, and (3) the excitation and emission of the NC. (b) Schematic of fast neutron imaging at the NECTAR beamline, with images of the scintillator enclosure and the NC samples which are sealed in a light-tight box. (c) Schematic of fast neutron imaging with colloidal NCs in cuvettes, and an averaged radiograph generated by the NC samples under five 60-s exposures to the fast neutron beam.

the meniscus of several of these colloidal NC solutions (e.g., Figure 2, Figure 4), which acts as a lens to redirect some of the light emitted away from the camera back toward it, these regions were excluded from analysis. Several images were also taken with a piece of a commercial $\mathrm{ZnS}: \mathrm{Cu}(\mathrm{PP})$ screen (e.g.,
Figure S5a), used here as a reference. Such screens were previously characterized on the same beamline. ${ }^{28}$ Concentration-dependence of the light yield was probed by diluting the fully concentrated samples in new cuvettes and taking additional radiographs, while thickness-dependence was 
a)

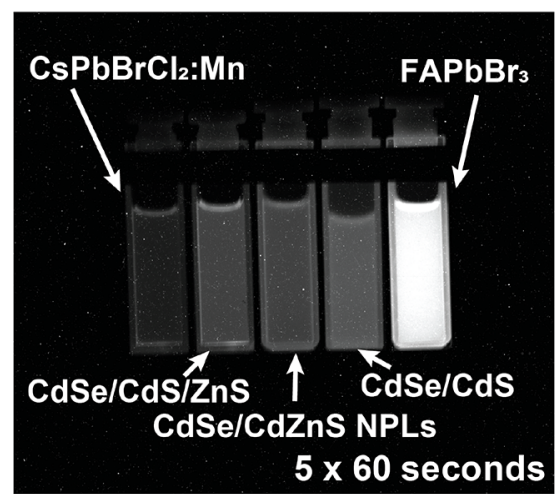

c)

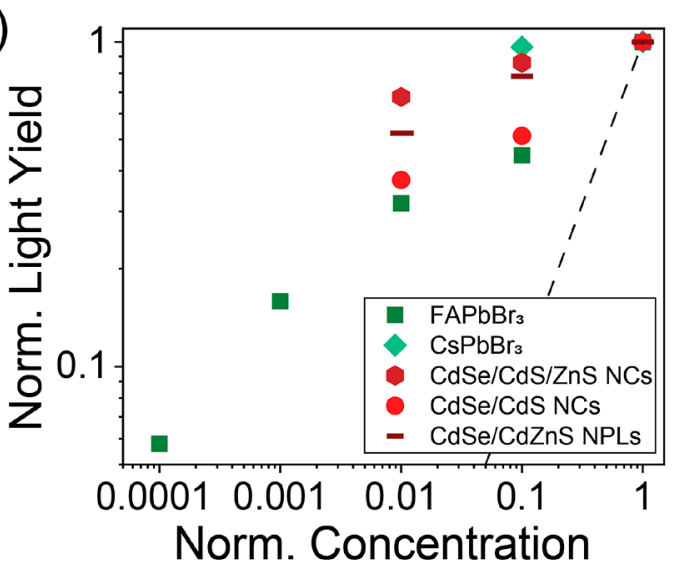

b)

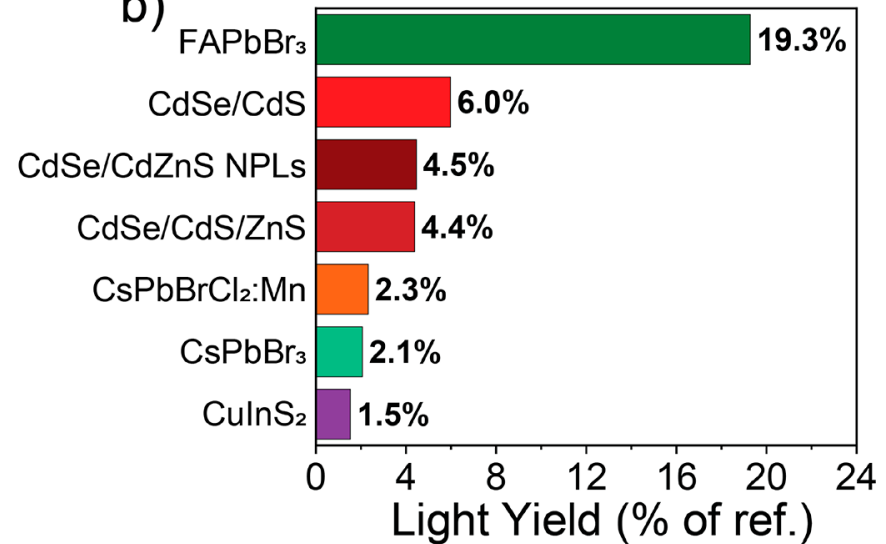

d)

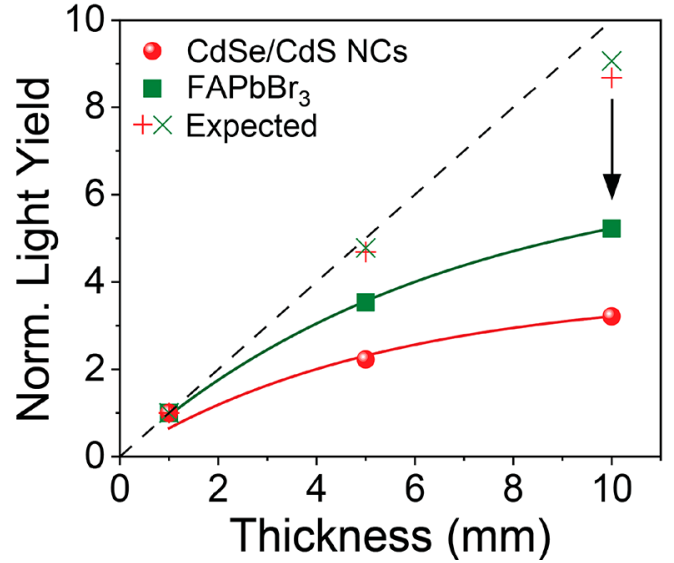

Figure 3. Total light yield comparisons of NC scintillators under fast neutron irradiation: (a) Radiograph of the five brightest NC emitters, average of five 60-s fast neutron exposures. (b) Bar graph of the highest light yield obtained for each NC emitter (at full concentrations as defined in Table 1, $10 \mathrm{~mm}$ thick cuvettes) under a 300-s fast neutron beam exposure, expressed as a percentage of the light yield obtained under identical conditions for the reference $\mathrm{ZnS}: \mathrm{Cu}(\mathrm{PP})$ scintillator screen. (c) Light yield vs concentration for each $\mathrm{NC}$ solution, 300-s exposure time. (d) Normalized light yield vs sample thickness for $\mathrm{FAPbBr}_{3}$ and $\mathrm{CdSe} / \mathrm{CdS} \mathrm{NCs}$ at full concentration, 300 -s exposure time, with cross and plus symbols representing the expected relative light yield due to the fast neutron scattering of each solvent at the given thickness and solid fit lines of the experimental light yield to eq 1 showing the reduction of light yield due to self-absorption.
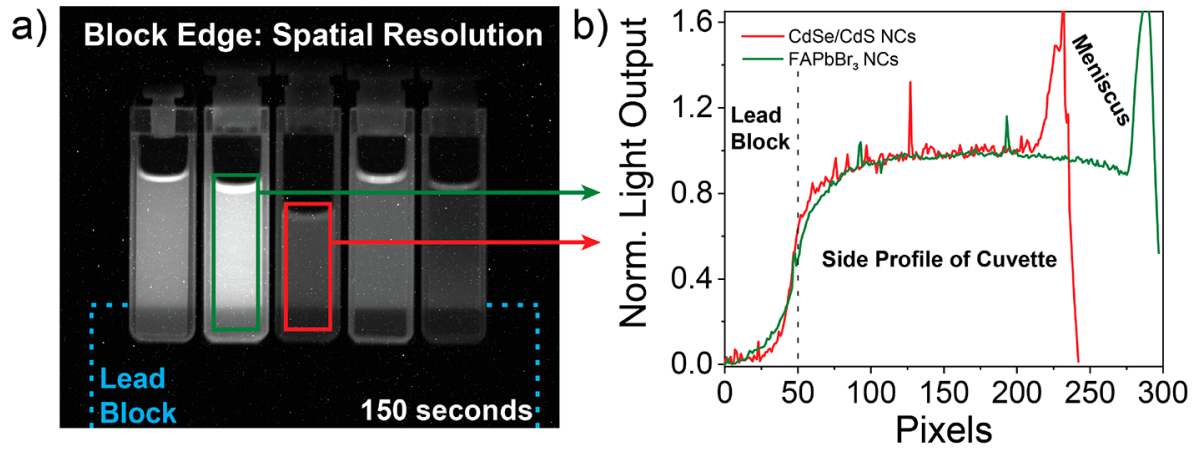

Figure 4. Spatial resolution of NC scintillators under fast neutron irradiation: (a) Radiograph of colloidal NCs with a lead block on the sample stage to provide a sharp edge to measure the ability of the NCs to resolve fine features, 150-s fast neutron beam exposure. (b) Emission intensity side profile of $\mathrm{FAPbBr}_{3}$ and $\mathrm{CdSe} / \mathrm{CdS} \mathrm{NC}$ cuvettes, with the lead block on the bottom of the cuvettes (pixel 0 ) and the meniscus waveguiding extra light out the top of the solution. (c) Bar graph of the spatial resolution obtained for each colloidal NC scintillator at full concentration (as defined in Table 1).

probed using cuvettes with variable thicknesses $(1,5$, and 10 $\mathrm{mm})$. The light yields of the $\mathrm{ZnS}: \mathrm{Cu}(\mathrm{PP})$ reference and all samples are found in Table S3.

All samples were measured for 30, 60, 120, and 300-s light yield exposures, with no significant deviations from linearity observed which would indicate the presence of afterglow effects or material degradation from fast neutron exposure
(Figure S2). To confirm the absence of long-lived luminescence afterglow on the order of seconds, each of the fully concentrated NC samples were exposed to the fast neutron beam for $10+$ minutes, then a 30 -s radiograph was measured immediately after the beam shutters were closed. The afterglow test radiographs showed no response from any of the NC samples (Figure S3c,d), confirming that these 
Table 1. Summary of NC Scintillator Material Characteristics for Fast Neutron Imaging

\begin{tabular}{|c|c|c|c|c|c|c|c|c|c|}
\hline $\mathrm{NC}$ emitter & $\begin{array}{l}\mathrm{NC} \sigma_{\gamma \text {-ray }} \\
\left(\mathrm{cm}^{2} / \mathrm{g}\right)\end{array}$ & solvent & $\begin{array}{c}\text { concn } \\
(\mathrm{mg} / \mathrm{mL})\end{array}$ & $a_{\gamma \text {-ray }}\left(10^{-3} \mathrm{~cm}^{-1}\right)$ & $\begin{array}{c}\text { PLQY } \\
(\%)\end{array}$ & $\begin{array}{l}\text { total light yield (\% } \\
\text { of } \mathrm{ZnS}: \mathrm{Cu}(\mathrm{PP}) \text { ref) }\end{array}$ & $\begin{array}{l}\text { spatial } \\
\text { resolution } \\
\text { (pixels) }\end{array}$ & $\begin{array}{l}\text { detected } \mathrm{n} / \gamma \text { - } \\
\text { ray ratio: } R_{\mathrm{n} / \gamma}\end{array}$ & $\begin{array}{l}\text { neutron light } \\
\text { yield (\% of } \\
\text { ref) }\end{array}$ \\
\hline $\mathrm{FAPbBr}_{3} \mathrm{NCs}$ & 0.28 & toluene & 18.4 & 5.2 & 96.2 & 19.28 & 52 & 3.7 & 30.4 \\
\hline $\mathrm{CdSe} / \mathrm{CdS} \mathrm{NCs}$ & 0.15 & hexane & 25 & 3.9 & 71.2 & 5.98 & 29 & 3.9 & 9.5 \\
\hline $\mathrm{CdSe} / \mathrm{CdZnS}$ NPLs & 0.17 & hexane & 10 & 1.7 & 70 & 4.46 & 36 & 2.7 & 6.5 \\
\hline $\mathrm{CdSe} / \mathrm{CdS} / \mathrm{ZnS} \mathrm{NCs}$ & 0.15 & cyclohexane & 11 & 1.5 & 65.6 & 4.39 & 35 & 2.8 & 6.5 \\
\hline $\mathrm{CsPbBrCl}_{2}: \mathrm{Mn} \mathrm{NCs}$ & 0.17 & hexane & 1.2 & 0.2 & 54.9 & 2.32 & 27 & 2.2 & 3.2 \\
\hline $\mathrm{Cs} \mathrm{PbBr}_{3} \mathrm{NCs}$ & 0.28 & dodecane & 34.9 & 9.8 & 32.3 & 2.07 & 41 & 4.1 & 3.3 \\
\hline $\mathrm{CuInS}_{2} \mathrm{NCs}$ & 0.14 & hexane & 40 & 5.7 & 70.3 & 1.53 & 35 & 2.4 & 2.2 \\
\hline
\end{tabular}

${ }^{a}$ Here $\sigma_{\gamma \text {-ray }}$ is the prompt ${ }^{235} U$ (vide infra) $\gamma$-ray cross section of each NC material, $a_{\gamma \text {-ray }}$ is the $\gamma$-ray attenuation coefficient accounting for the NC concentration of the NC solution, $R_{\mathrm{n} / \gamma}$ is the ratio of neutron-induced to $\gamma$-ray-induced radioluminescence, and the neutron-specific light yield is obtained from the total light yield by taking into account $R_{\mathrm{n} / \gamma}$.

materials exhibit decay times below the order of seconds. This is an important advantage over the $\mathrm{ZnS}: \mathrm{Cu}(\mathrm{PP})$ screens, which exhibit afterglow effects on the order of minutes. ${ }^{28}$

The light yield of each scintillator under identical measurement conditions ( $10 \mathrm{~mm}$ cuvettes, 300 -s fast neutron exposure, and at full concentration as defined in Table 1) is found in Figure $3 b$, expressed as a percentage of the response of the $\mathrm{ZnS}: \mathrm{Cu}(\mathrm{PP})$ reference screen. The perovskite $\mathrm{FAPbBr}_{3}$ is the brightest material among the tested samples by a factor of 3 at $19.3 \%$, followed by the CdSe-based nanomaterials, exhibiting $4.4 \%$ to $6 \%$ of the light yield of the $\mathrm{ZnS}: \mathrm{Cu}(\mathrm{PP})$ reference. The doped perovskite $\mathrm{CsPbBrCl} 2: \mathrm{Mn}$ emitted $2.3 \%$, while $\mathrm{Cs} \mathrm{PbBr}_{3}$ and $\mathrm{CuInS}_{2}$ only emit $2.1 \%$ and $1.5 \%$ of the reference, respectively.

It is important to note that we did not normalize to any of the relevant parameters that dictate the light yield (e.g., hydrogen density of the solvent, PLQY of the sample, the sample thickness, or the concentration/amount of material) as each material is different and cannot necessarily be prepared with the same parameters as another. The latter factor is especially important to consider as the $\mathrm{ZnS}: \mathrm{Cu}(\mathrm{PP})$ screen contains 30 weight $\% \mathrm{ZnS}: \mathrm{Cu}$, while the colloidal $\mathrm{FAPbBr}_{3}$ solution only contains about 2 weight $\% \mathrm{FAPbBr}_{3} \mathrm{NCs}$. Given the number of tunable factors which have not been optimized, at this stage the actual value achieved is the most direct and reliable measure of the potential of these materials, with further improvements in light yield expected as the community learns how to tune these parameters to achieve the brightest scintillators. On the basis of these results, the brightest materials were selected to study the effect of different concentrations and thicknesses.

The light yield increases with higher concentration for all five samples tested, but the increase is far from linear as shown by the dotted line in Figure 3c. The light yield of $\mathrm{FAPbBr}_{3}$ only increases by a factor of 17 over 4 orders of magnitude increase in the concentration, while those of the CdSe-based materials vary by a factor of 3 over a 2 orders of magnitude increase in concentration, and the light yield of $\mathrm{CsPbBr}_{3}$ is nearly constant. There are two primary concentration-dependent properties that compete to yield this complex behavior: charge conversion efficiency and self-absorption. Higher concentration gives more NCs per volume, yielding a higher CCE as it becomes more probable that proton-ionized charges are close enough to excite NC emission. This will improve the light yield as concentration rises, with saturation at some point as the phosphor loading is sufficient to drive the CCE to unity. On the other hand, self-absorption (that is, reabsorption of the NC radioluminescence by neighboring NCs) will decrease the light yield at high concentrations as the solution gains optical density and becomes more likely to reabsorb the emitted photons. The photon is then emitted again with the average probability equal to the PLQY of the colloid. Multiple rounds of such a photon recycling diminish the overall light output. In the case of $\mathrm{CsPbBr}_{3} \mathrm{NC}$ colloid with its lower PLQY and high concentration ( $32.3 \%$ and $34.9 \mathrm{mg} / \mathrm{mL}$, respectively), increased self-absorption almost cancels out the improved CCE going from the $1 / 10$ diluted to the fully concentrated solution. The concentration-dependent light yield of $\mathrm{FAPbBr}_{3}$ and the CdSe-based materials each continue to increase, indicating that the improved CCE outweighs any increase in self-absorption and that each solution lies below the "ideal" concentration for maximum light output.

The thickness-dependence of the light yield in $\mathrm{CdSe} / \mathrm{CdS}$ and $\mathrm{FAPbBr}_{3} \mathrm{NCs}$ deviates significantly both from linearity (dotted line) as well as the trend calculated from the fast neutron scattering in each solvent as given by the BeerLambert law (cross and plus symbols calculated by the fast neutron stopping power of the solvents toluene and hexane, respectively, Figure $3 \mathrm{~d}$ ). This deviation can be the result of either a saturation of fast neutron attenuation, or the presence of enhanced self-absorption with higher thickness, as emitted light on average must traverse a farther distance within the absorptive medium. For a material with neutron attenuation $a$ and absorption attenuation coefficient $b$, we integrate the probability of neutron attenuation before the NC interaction and light absorption between the NC emission and the end of the optical path in the cuvette for every distance $x$ in the material over the thickness $d$, yielding the following thicknessdependent light yield:

$$
\text { L. Y. }=C \int_{0}^{d} \mathrm{e}^{-a x} \mathrm{e}^{-b(d-x)} \mathrm{d} x=\frac{C}{a-b}\left(\mathrm{e}^{-a d}-\mathrm{e}^{-b d}\right)
$$

This equation fits the light yield of $\mathrm{FAPbBr}_{3}$ and $\mathrm{CdSe} / \mathrm{CdS}$ NCs well (solid lines, Figure $3 \mathrm{~d}$ ) with the neutron attenuation coefficient $a$ refining to 0 , indicating that only increased light absorption is responsible for reducing the light yield in these materials. This is consistent with the low attenuation coefficient of fast neutrons even in hydrogen-rich materials such as hexane (only $22.8 \%$ of fast neutrons scatter in a cmthick solution of hexane), so neutron attenuation is far from saturation and thicker samples will enhance light yield by producing more recoil proton generation events. Thus, it is clear that self-absorption is the limiting factor that reduces the actual light yield to $58 \%$ and $37 \%$ of the expected value for $\mathrm{FAPbBr}_{3}$ and $\mathrm{CdSe} / \mathrm{CdS} \mathrm{NCs}$, respectively. 
a)

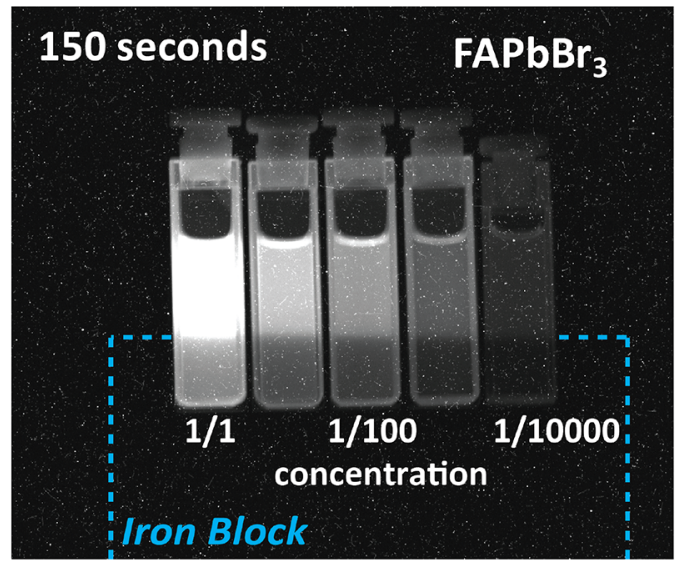

c)

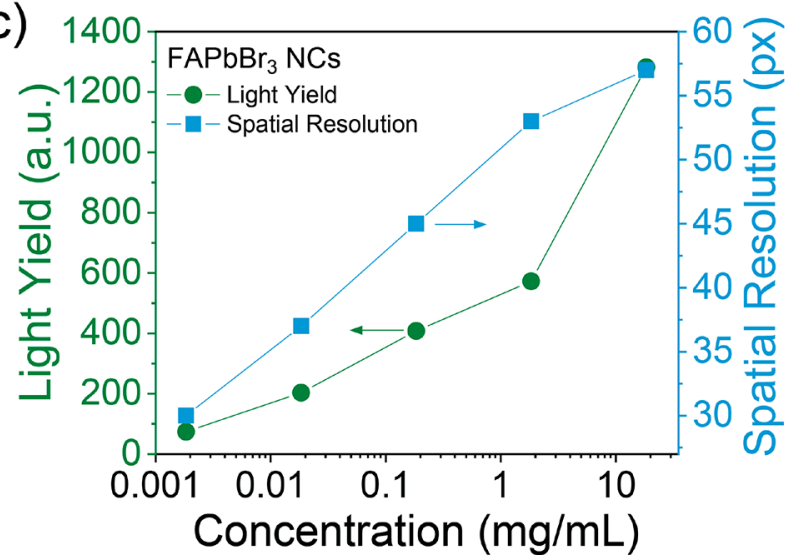

b)
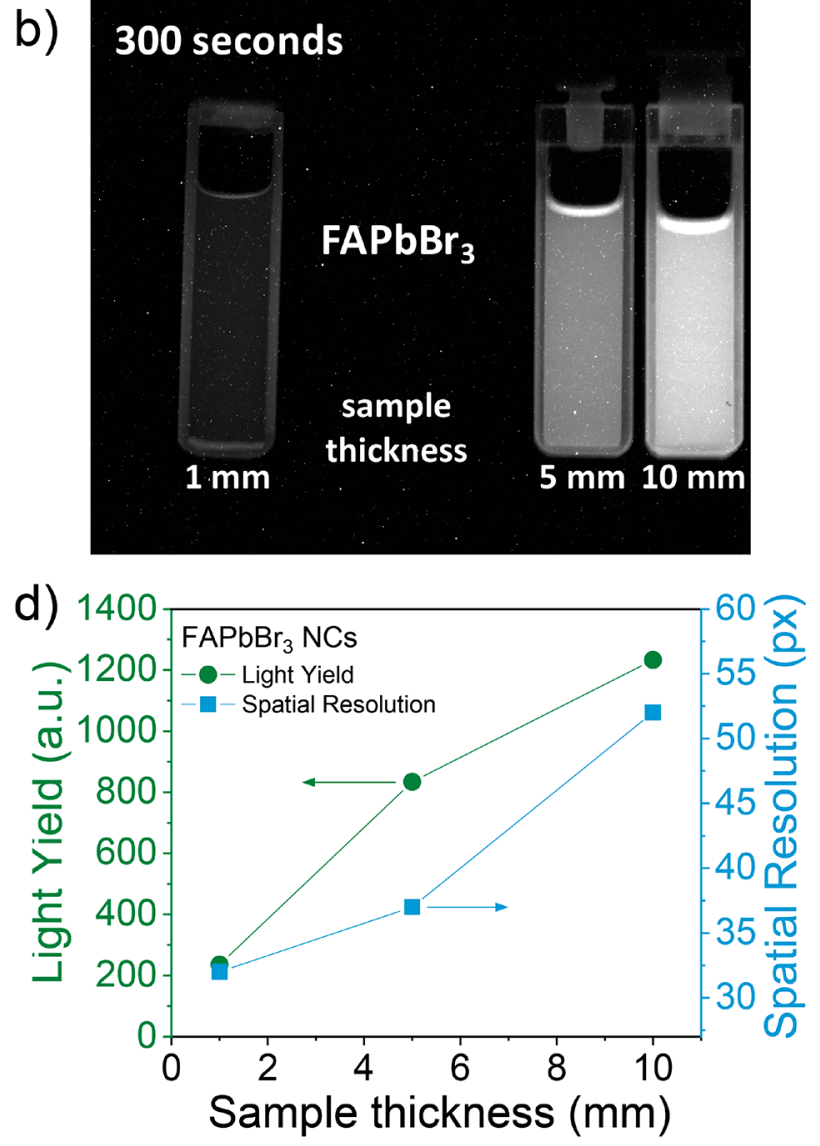

Figure 5. $\mathrm{FAPbBr}_{3}$ concentration and thickness-dependence: (a) Radiograph with iron block showcasing spatial resolution of FAPbBr $\mathrm{NCs}_{3}$ vs concentration, 150-s fast neutron beam exposure. (b) Radiograph of different thickness $\mathrm{FAPbBr}_{3}$ samples, 300 -s fast neutron exposure. Light yield and spatial resolution (in pixels) as a function of (c) concentration and (d) sample thickness.

Now that the concentration-dependence is understood, we can revisit the above results and more accurately compare different materials. The CdSe-based nanomaterials exhibit similar PLQYs from 65 to $71 \%$ in solvents with similar hydrogen density (hexane and cyclohexane), and accordingly we observe that $\mathrm{CdSe} / \mathrm{CdS} \mathrm{NCs}$ had the highest light yield $(6.0 \%)$ due to their higher concentration $(25 \mathrm{mg} / \mathrm{mL})$ over the $\mathrm{CdSe} / \mathrm{CdS} / \mathrm{ZnS} \mathrm{NCs}$ and $\mathrm{CdSe} / \mathrm{CdZnS}$ NPLs which had light yields and concentrations of $4.4 \%$ and $4.5 \%$ and 11 and $10 \mathrm{mg} /$ $\mathrm{mL}$, respectively. Hence, the effects of morphology and core/ shell structure are not so significant here because each material exhibits similar PLQY. We conclude that these features do not obviously affect the CCE.

The low light yield of core/shell $\mathrm{CuInS}_{2} / \mathrm{ZnSe} / \mathrm{ZnS} \mathrm{NCs}$ was rather unexpected given the high PLQY and Stokes shift $(150 \mathrm{meV})$ for this material; ${ }^{55}$ however, this does not account for the breadth of the emission and the absorption of the $\mathrm{CuInS}_{2}$ core with its $1.5 \mathrm{eV}$ band gap ${ }^{56}$ which lead to significant self-absorption even at $700 \mathrm{~nm}$, below the $668 \mathrm{~nm}$ peak energy (Figure S1d). This self-absorption and the high concentration are responsible for the poor light yield of $\mathrm{CuInS}_{2}$ compared to the other NCs tested here.

In contrast to the similar performance of the CdSe-based materials, the perovskites exhibited variable performance with the hybrid organic-inorganic $\mathrm{FAPbBr}_{3}$ far outpacing the inorganic perovskites at $2.3 \%$ for $\mathrm{CsPBrCl}_{2}: \mathrm{Mn}$ and $2.1 \%$ for $\mathrm{CsPbBr}_{3}$. The higher light yield of $\mathrm{CsPbBrCl}_{2}: \mathrm{Mn}$ over $\mathrm{CsPbBr}{ }_{3}$ despite the vastly higher concentration of the latter material $(1.2 \mathrm{mg} / \mathrm{mL}$ to $34.9 \mathrm{mg} / \mathrm{mL})$ highlights the benefits of Stokes-shifted emission which minimizes self-absorption. The undoped perovskites provide an instructive case for the importance of self-absorption, as the PLQY of $\mathrm{FAPbBr}_{3}$ is roughly three times that of $\mathrm{CsPBr}_{3}$ while the light yield was 9.3 times higher. The remaining factor of 3 is caused by the smaller Stokes shift of $\mathrm{CsPbBr}_{3}$ which leads to an increased overlap of the emission and absorption spectra (Figure S4a,c). Integrating these overlaps to find the self-absorption coefficient for each material, the path of photons in both perovskites was simulated using this coefficient with the experimentally determined PLQYs, sample photon tracks are shown in Figure $\mathrm{S} 4 \mathrm{~b}, \mathrm{~d}$. The simulations predict a ratio of light yield in $\mathrm{FAPbBr}_{3}$ to $\mathrm{CsPbBr}_{3}$ of 9.8 , in excellent agreement with the experimental results (ratio of 9.3) and confirming the detrimental self-absorption of $\mathrm{CsPbBr}_{3}$ as the cause of its lower light yield.

These trends reveal the detrimental effect of self-absorption on the light yield of the NC scintillators, and also indicate that higher concentrations on the order of $10 \mathrm{wt} \%$ would be desirable to give a higher density of NCs in solution and increase the CCE. Despite these factors, these NC materials demonstrate excellent response to recoil protons, with the light yield of $\mathrm{FAPbBr}_{3}$ only a factor of 5 less than that of the commercial $\mathrm{ZnS}: \mathrm{Cu}(\mathrm{PP})$ screen despite having much smaller phosphor load. Note also that the light output of $\mathrm{ZnS}: \mathrm{Cu}(\mathrm{PP})$ is overestimated here because of the effects of build-up from gamma-induced afterglow of the $\mathrm{ZnS}: \mathrm{Cu}(\mathrm{PP})^{28}$ and benefits 
further from its higher gamma sensitivity relative to these NC materials (vide infra). In contrast, the lack of long-lived afterglow in the NC materials is promising for the use of these materials as screens for computed tomography which require many scans in rapid succession.

Spatial Resolution. The spatial resolution of the NC scintillators was estimated by placing a thick metal block (iron, lead, or tungsten) on the sample stage to give a sharp edge across the cuvettes (e.g., Figure 4a, where the lead block is marked with a dotted blue line). The averaged side profile of the cuvette was normalized to the low and high light yield portions of the cuvette, corresponding to the blocked and unblocked fast neutron beam, respectively (Figure $4 \mathrm{~b}$ ). The spatial resolution is estimated using the pixel width of the edge as given by the $10 \%-90 \%$ rise distance, demonstrated in Figure S5 for the commercial $\mathrm{ZnS}: \mathrm{Cu}(\mathrm{PP})$ screen to obtain a resolution of 22 pixels. In the present camera configuration and focus, each pixel corresponds to a width of approximately 0.1 $\mathrm{mm}$, as measured by the width of the cuvettes (103 pixels for $10 \mathrm{~mm}$, or $0.097 \mathrm{~mm} /$ pixel).

The spatial resolution for each NC scintillator in $10 \mathrm{~mm}$ cuvettes measured at full concentration (as given in Table 1) using 5 averaged 60-s fast neutron exposures is given in Figure 4c. The best value ( 27 pixels, $\sim 2.7 \mathrm{~mm}$ ) is obtained for the doped perovskite $\mathrm{CsPbBrCl}_{2}: \mathrm{Mn}$, while the highest edge widths of 41 and 52 pixels are obtained for the perovskites $\mathrm{CsPbBr}$ and $\mathrm{FAPbBr}_{3}$, respectively. The CdSe-based nanomaterials and $\mathrm{CuInS}_{2} \mathrm{NCs}$ have intermediate spatial resolutions ranging from 29 to 36 pixels. Similar to the light yield, these values can be largely understood as a function of the self-absorption of these materials, as extraneous absorption and re-emission will blur the edges. $\mathrm{CsPbBrCl}_{2}: \mathrm{Mn}$ with its highly Stokes-shifted $\mathrm{Mn}$ dopant emission ${ }^{57}$ thus exhibits the sharpest resolution, while the low Stokes-shift perovskites exhibit the broadest edge widths. The high edge width of core/ shell $\mathrm{CuInS}_{2} / \mathrm{ZnSe} / \mathrm{ZnS} \mathrm{NCs}$ is likely due to the significant self-absorption of the $\mathrm{CuInS}_{2}$ core, as described above. The vastly higher edge width for $\mathrm{FAPbBr}_{3}$ of 52 pixels (over double the reference screen at 22 pixels) stands out, and potentially could be attributed to more efficient photon recycling ${ }^{58,59}$ (due to the high PLQY of this sample) which would enhance the absorption and re-emission that smears the sharp edge.

The spatial resolution improves for samples with reduced sample thickness and concentration, as shown in Figure 5 for $\mathrm{FAPbBr}_{3}$. However, the light yield also decreases rapidly with reduced thickness and concentration (Figure 5c,d), forcing a compromise between good spatial resolution and high light yield. The drastic falloff of light yield due to concentration in $\mathrm{FAPbBr}_{3}$ again highlights the effects of self-absorption, as this trade-off would be less severe in a material with a high Stokes shift. Such thickness-dependence is common with scintillation detectors, as thickness is required for higher stopping power (and therefore higher detection efficiency) while high thicknesses often reduce the spatial resolution, similar effects in fast neutron imaging were observed for commercial $\mathrm{ZnS}: \mathrm{Cu}(\mathrm{PP})$ and $\mathrm{ZnS}: \mathrm{Ag}(\mathrm{PP})$ screens. $^{28}$

Neutron and $\gamma$-ray Sensitivity. Because uranium fission is used as the fast neutron source, the radiation beam includes a high $\gamma$-ray flux as well as fast neutrons. ${ }^{60}$ Therefore, it is important to evaluate the potential contribution of $\gamma$-rays to the observed NC scintillation. The calculated $\gamma$-ray attenuation cross sections $\sigma_{\gamma \text {-ray }}$ of the NCs scintillator materials, averaged with respect to the prompt gamma ray spectrum of ${ }^{235} U,{ }^{5,61}$ range from $0.14 \mathrm{~cm}^{2} / \mathrm{g}$ in $\mathrm{CuInS}_{2}$ to $0.28 \mathrm{~cm}^{2} / \mathrm{g}$ for $\mathrm{FAPbBr}_{3}$ and $\mathrm{CsPbBr}_{3}$ (Table 1), consistent with the photon stopping power provided by the high $\mathrm{Pb}$ content of the latter compounds. The attenuation coefficients $a_{\gamma \text {-ray }}$ of these NC samples, which account for the concentration by dividing by the NC density in the colloidal solution, vary from $0.2 \times 10^{-3}$ $\mathrm{cm}^{-1}$ for the more dilute $\mathrm{CsPbBrCl}_{2}: \mathrm{Mn} \mathrm{NCs}$ to $9.8 \times 10^{-3}$ $\mathrm{cm}^{-1}$ for the more concentrated $\mathrm{CsPbBr}_{3}$ NCs (Table 1). These values are quite low, corresponding to attenuation lengths of approximately 5000 and $100 \mathrm{~cm}$, respectively, reflecting a low detection efficiency of $\gamma$-rays because the density of NCs in solution is low compared to a bulk material.

The blocking experiment implemented for the spatial resolution provides a modulated light yield due to neutron and gamma attenuation of the metal blocks, thus the ratio $R_{\mathrm{b}}$ of the light intensity in the blocked and unblocked regions can be used to estimate the gamma sensitivity. This ratio can be expressed as

$$
R_{\mathrm{b}}=\frac{N_{n_{\text {blocked }}}+N_{\gamma_{\text {blocked }}}}{N_{n_{\text {light }}}+N_{\gamma_{\text {light }}}}=\frac{N_{n_{\text {light }}} \mathrm{e}^{-d a_{n}}+N_{\gamma_{\text {light }}} \mathrm{e}^{-d a_{\gamma}}}{N_{n_{\text {light }}}+N_{\gamma_{\text {light }}}}
$$

where $N_{\gamma}$ and $N_{n}$ are the number of scintillation photons induced by $\gamma$-rays and neutrons, respectively, $d$ is the block thickness, and $a_{n}$ and $a_{\gamma}$ are the attenuation coefficients of neutrons and $\gamma$-rays in the metal block. Rewriting (2), we can find the ratio $R_{n / \gamma}$ of light yield induced by neutrons and $\gamma$-rays in terms of $R_{\mathrm{b}}$ and the block attenuation coefficients:

$$
R_{n / \gamma}=\frac{N_{n_{\text {light }}}}{N_{\gamma_{\text {light }}}}=\frac{\mathrm{e}^{-d a_{\gamma}}-R_{\mathrm{b}}}{R_{\mathrm{b}}-\mathrm{e}^{-d a_{n}}}
$$

Gamma attenuation coefficients of the metal blocks were averaged with respect to the ${ }^{235} U \gamma$-ray spectrum, ${ }^{61}$ while the fast neutron attenuation coefficients were estimated using the calculated neutron fission spectrum. ${ }^{11}$ Note that these values will overestimate the neutron stopping power due to the lower average energy of the calculated fission neutron spectrum $(1.35$ $\mathrm{MeV})$ versus the actual fast neutron beam $\left(1.8 \mathrm{MeV}^{53}\right)$, thereby underestimating the neutron contribution to the light yield.

The light yield ratio $R_{n / \gamma}$ ranges from 2.2 in $\mathrm{CsPbBrCl}_{2}: \mathrm{Mn}$ NCs to 4.1 in $\mathrm{FAPbBr}_{3}$, showing that while the majority of the emission is neutron-induced, the light yield fraction induced by $\gamma$-rays $(19.6 \%-31.0 \%)$ is non-negligible despite the low detection efficiency expected for NCs. Even considering the underestimation of the neutron contribution, it is clear that a substantial portion (approximately 10-20\%) of the light yield is induced by what must be a high flux of $\gamma$-rays.

These values compare favorably to $\mathrm{ZnS}: \mathrm{Cu}(\mathrm{PP})$, which has a reported ratio of 1.0-1.1, while matching or surpassing the performance of $\mathrm{ZnS}: \mathrm{Ag}(\mathrm{PP})$ with a $R_{n / \gamma}$ of $1.7-3.3$, both measured on this same beamline. ${ }^{28}$ This also implies that the light yield of $\mathrm{ZnS}: \mathrm{Cu}(\mathrm{PP})$ is overestimated relative to our $\mathrm{NC}$ materials due to its higher $\gamma$-ray sensitivity. Using these ratios to obtain the neutron-specific light yield, we find that the neutron-generated light yields range from $2.2 \%$ of the ref $\mathrm{ZnS}: \mathrm{Cu}(\mathrm{PP})$ for $\mathrm{CuInS}_{2}$ to $30.4 \%$ for $\mathrm{FAPbBr}_{3}$ (Table 1).

These values have important practical implications because $\gamma$-rays always contaminate fast neutron beams, and often extensive sets of filters are required to remove these undesired rays, at the cost of weakening the fast neutron flux as well. The low $R_{n / \gamma}$ of $\mathrm{ZnS}: \mathrm{Cu}(\mathrm{PP})$ means at least $14 \mathrm{~cm}$ of $\mathrm{Pb}$ is required 
to bring the $\gamma$-ray contribution down to $1 \%$ of the signal, while only $6.8 \mathrm{~cm}$ was needed for a $R_{n / \gamma}$ of $3.3 .{ }^{28}$ Hence, while filtering the gamma signal with additional $\mathrm{Pb}$ filters would still be necessary for fast neutron imaging, significantly less material would be required to bring the $\gamma$-ray contribution down for $\mathrm{FAPbBr}_{3} \mathrm{NCs}\left(R_{n / \gamma}\right.$ of 3.7), permitting operation under a higher fast neutron flux. Higher $R_{n / \gamma}$ is particularly important in tomography applications in which removal of the additional $\gamma$ ray induced "background" signal is infeasible in calculationheavy reconstruction tasks.

\section{CONCLUSIONS}

We have demonstrated fast neutron imaging using $\mathrm{NC}$ scintillators in organic solvent as recoil proton detectors and compared a variety of halide and chalcogenide NC systems. $\mathrm{FAPbBr}_{3} \mathrm{NCs}$ exhibited the highest light output (with $30.4 \%$ neutron-specific light yield relative to that of the $\mathrm{ZnS}: \mathrm{Cu}(\mathrm{PP})$ reference) despite being in the least hydrogen-dense solvent, while $\mathrm{NCs}$ of the doped perovskite $\mathrm{CsPbBrCl}$ : $\mathrm{Mn}$ had the best spatial resolution. The NC emitters exhibited significantly lower $\gamma$-ray specific sensitivity than $\mathrm{ZnS}: \mathrm{Cu}(\mathrm{PP})$ screens and uniformly lacked afterglow, potentially making them suitable for applications requiring fast response times. Concentration and thickness-dependence of the light yield and spatial resolution revealed the key parameters to optimize, yielding the following design principles for NC-based recoil proton detectors:

1. Low self-absorption is required to ensure that the materials are transparent to their own emission, thereby reducing the loss of light yield and resolution from large thicknesses and high concentration. Hence materials with high Stokes shifts are desired.

2. High concentrations $(>20 \mathrm{mg} / \mathrm{mL})$ are needed to ensure that there are enough NCs per volume to achieve high conversion efficiencies.

3. High PLQY is needed for NCs and proton-rich solvents to maximize charge conversion and neutron interaction efficiencies.

Although these initial results have not yet exceeded the commercial standard's light output and spatial resolution, NCs following the above design principles promise to substantially enhance performance in both aspects, while maintaining the advantages of significantly lower decay time and reduced gamma sensitivity. Beyond simple optimization, important open questions remain to be explored, such as the effect of NC size or $\mathrm{H}$-containing $\mathrm{NCs}$ on light yield, or the potential charge conversion efficiency of differently capped NCs on recoil detection performance. Further investigation of these factors are planned for upcoming run-cycles of the FRM-II. These results enable the development of NC recoil proton detectors in fast neutron imaging that can potentially transform the field and foster widespread application of this technique to a variety of problems inaccessible to other radiation sources.

\section{METHODS}

Optical Characterization. Absorption and photoluminescence spectroscopy were performed for each colloidal nanocrystal solution sample at room temperature. The samples were prepared typically by diluting $20 \mu \mathrm{L}$ of nanocrystal solution in $2 \mathrm{~mL}$ of the corresponding solvent. This diluted solution was transferred to a $1 \mathrm{~cm}$ quartz cuvette for the optical measurements. UV-vis absorption measurements were conducted using a Jasco V670 (V770 for $\mathrm{FAPbBr}_{3} \mathrm{NCs}$ ) spectrometer in transmission mode. Steady-state PL spectra from the solutions were measured using a Fluorolog iHR 320 Horiba Jobin Yvon spectrofluorometer equipped with a PMT detector (HoribaFluoromax-4P+ equipped with a PMT detector in the case of $\mathrm{FAPbBr}_{3}$ $\mathrm{NCs}$ ) and a monochromatized $150 \mathrm{~W}$ xenon lamp as an excitation source. Spectra were corrected to account for the spectral sensitivity of the setup. Absolute PLQY of NC solutions was measured using a Quantaurus-QY Absolute PL quantum yield spectrometer from Hamamatsu.

Fast Neutron Imaging at NECTAR. Fast neutron imaging was conducted at the NECTAR (NEutron Computed Tomography And Radiography) beamline of the FRM-II reactor in Garching, Germany, ${ }^{53}$ under their user program. A schematic of the instrument is found in Figure $2 \mathrm{~b}$. The setup is described more thoroughly in the main text and consists of the beamline components, a sample stage to place materials for imaging, and a light-tight scintillator enclosure with a CCD camera (CCD ANDOR iKon-L BV camera). ${ }^{54}$ The camera was cooled to $-100{ }^{\circ} \mathrm{C}$ and has an active area of $27.6 \mathrm{~mm} \times 27.6 \mathrm{~mm}$, with a $2048 \times 2048$ pixel grid $(13.5 \mu \mathrm{m} \times 13.5 \mu \mathrm{m}$ each $)$. The collimation ratio, $L / D$, at NECTAR was set to 200 using a recently upgraded revolver collimator system.

To test the neutron attenuation of the NC samples, cuvettes of each colloidal NC material at full concentration are placed on the sample stage while the commercial $\mathrm{ZnS}$ : $\mathrm{Cu}$ reference screen is used as the fast neutron scintillator in the enclosure. Three 30-s radiographs were taken of each set of cuvettes, and the sum of these frames was used to estimate the neutron attenuation of the colloidal NCs.

Following these tests, the reference screen was removed from the scintillator enclosure and replaced by a thin aluminum plate with a 5cuvette sample holder. The colloidal NC scintillator materials were secured to the sample holder (which had plastic slots to isolate the cuvettes from exciting each other), and the aluminum plate was sealed onto the light-tight box with aluminum tape. Radiographs for light yield were taken without any objects on the sample stage, while those for spatial resolution measurements were taken with thick metal blocks (either W with $1.5 \mathrm{~cm}$ thickness, Fe with $5 \mathrm{~cm}$ thickness, or a $2.5 \mathrm{~cm}$-thick $\mathrm{Pb}$ block) in the beam path to provide a sharp edge.

Samples were diluted in a nearby facility following the results of the first frames, in order to optimize the use of beamtime and due to the inability to reuse cuvettes once exposed. (They require checking by the Radiation Safety department, which generally takes place once per day, to ensure that the samples are not activated by the neutron imaging beam. This should be considered when planning similar experiments.)

Images were processed using the Fiji distribution ${ }^{62}$ of Image ${ }^{63}$ permitting the settings to be tuned for best visibility of each radiograph. The area selector tool was used to measure the light output for selected regions in and around the cuvette for light yield comparisons, and the plot profile function was used to obtain a side profile of the cuvettes for spatial resolution fitting. Note that the light intensity for all measured frames was well below the saturation point of the CCD camera, ensuring that the light response is linear to light yield for all samples.

Simulation of Self-Absorption Influence on Light Yield. The simulations were implemented in the Geant 4 software package. ${ }^{64}$ The cuvettes were simulated with the real dimensions, and the optical properties (absorption length, re-emission probability) of the corresponding NC solutions were assigned to this volume to take into account self-absorption and possible photon recycling. The camera was simulated by a photon detection plate on the side of the volume. Owing to the low attenuation of fast neutrons in the experiments, the simulation photons were generated homogeneously in the cuvette volume, with the number of generated photons proportional to the neutron attenuation of the particular solvent of each NC. These photons were assigned random directions and energies corresponding to the emission spectrum of the scintillator. The number of detected photons was calculated and compared for $\mathrm{FAPbBr}_{3}$ and $\mathrm{CsPbBr}_{3}$ NCs. 


\section{ASSOCIATED CONTENT}

\section{(1) Supporting Information}

The Supporting Information is available free of charge at https://pubs.acs.org/doi/10.1021/acsnano.0c06381.

Experimental details of nanocrystal synthesis, absorption and PL spectra, afterglow test radiographs, light yield vs time plot, spatial resolution of $\mathrm{ZnS}: \mathrm{Cu}(\mathrm{PP})$ reference, comparison of self-absorption in $\mathrm{CsPbBr}_{3}$ and $\mathrm{FAPbBr}_{3}$. (PDF)

\section{AUTHOR INFORMATION}

\section{Corresponding Author}

Maksym V. Kovalenko - Laboratory of Inorganic Chemistry, Department of Chemistry and Applied Biosciences, ETH Zürich, Zürich CH-8093, Switzerland; Laboratory for Thin Films and Photovoltaics, Empa-Swiss Federal Laboratories for Materials Science and Technology, Dübendorf CH-8600, Switzerland; ๑ orcid.org/0000-0002-6396-8938; Email: mvkovalenko@ ethz.ch

\section{Authors}

Kyle M. McCall - Laboratory of Inorganic Chemistry, Department of Chemistry and Applied Biosciences, ETH Zürich, Zürich CH-8093, Switzerland; Laboratory for Thin Films and Photovoltaics, Empa-Swiss Federal Laboratories for Materials Science and Technology, Dübendorf CH-8600, Switzerland; (ㄱ) orcid.org/0000-0001-8628-3811

Kostiantyn Sakhatskyi - Laboratory of Inorganic Chemistry, Department of Chemistry and Applied Biosciences, ETH Zürich, Zürich CH-8093, Switzerland; Laboratory for Thin Films and Photovoltaics, Empa-Swiss Federal Laboratories for Materials Science and Technology, Dübendorf CH-8600, Switzerland; (1) orcid.org/0000-0003-2384-1665

Eberhard Lehmann - Paul Scherrer Institut, Villigen PSI 5232, Switzerland; (i) orcid.org/0000-0001-9145-9009

Bernhard Walfort - RC Tritec Ltd., Teufen 9053, Switzerland

Adrian S. Losko - Forschungs-Neutronenquelle Heinz Maier-

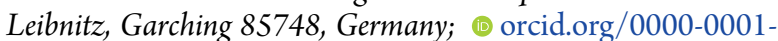
5307-356X

Federico Montanarella - Laboratory of Inorganic Chemistry, Department of Chemistry and Applied Biosciences, ETH Zürich, Zürich CH-8093, Switzerland; Laboratory for Thin Films and Photovoltaics, Empa-Swiss Federal Laboratories for Materials Science and Technology, Dübendorf CH-8600, Switzerland; (1) orcid.org/0000-0002-9057-7414

Maryna I. Bodnarchuk - Laboratory of Inorganic Chemistry, Department of Chemistry and Applied Biosciences, ETH Zürich, Zürich CH-8093, Switzerland; Laboratory for Thin Films and Photovoltaics, Empa-Swiss Federal Laboratories for Materials Science and Technology, Dübendorf CH-8600, Switzerland; (1) orcid.org/0000-0001-6597-3266

Franziska Krieg - Laboratory of Inorganic Chemistry, Department of Chemistry and Applied Biosciences, ETH Zürich, Zürich CH-8093, Switzerland; Laboratory for Thin Films and Photovoltaics, Empa-Swiss Federal Laboratories for Materials Science and Technology, Dübendorf CH-8600, Switzerland; (1) orcid.org/0000-0002-0370-1318

Yusuf Kelestemur - Laboratory of Inorganic Chemistry, Department of Chemistry and Applied Biosciences, ETH Zürich, Zürich CH-8093, Switzerland; Laboratory for Thin Films and Photovoltaics, Empa-Swiss Federal Laboratories for Materials Science and Technology, Dübendorf CH-8600, Switzerland;
Department of Metallurgical and Materials Engineering, Atilim University, Ankara 06830, Turkey; 이이이.org/0000-00031616-2728

David Mannes - Paul Scherrer Institut, Villigen PSI 5232, Switzerland; (1) orcid.org/0000-0003-4028-7504

Yevhen Shynkarenko - Laboratory of Inorganic Chemistry, Department of Chemistry and Applied Biosciences, ETH Zürich, Zürich CH-8093, Switzerland; Laboratory for Thin Films and Photovoltaics, Empa-Swiss Federal Laboratories for Materials Science and Technology, Dübendorf $\mathrm{CH}-8600$, Switzerland; (C) orcid.org/0000-0002-1587-1752

Sergii Yakunin - Laboratory of Inorganic Chemistry, Department of Chemistry and Applied Biosciences, ETH Zürich, Zürich CH-8093, Switzerland; Laboratory for Thin Films and Photovoltaics, Empa-Swiss Federal Laboratories for Materials Science and Technology, Dübendorf CH-8600, Switzerland; (1) orcid.org/0000-0002-6409-0565

Complete contact information is available at:

https://pubs.acs.org/10.1021/acsnano.0c06381

\section{Notes}

The authors declare no competing financial interest.

\section{ACKNOWLEDGMENTS}

This work was financially supported by RC Tritec AG and by ETH Zurich through the ETH+ Project SynMatLab. F.M. acknowledges support by ETH through the ETH Postdoctoral Fellowship. This work is based upon experiments performed at the NECTAR instrument operated by FRM II at the Heinz Maier-Leibnitz Zentrum (MLZ), Garching, Germany. The authors acknowledge fruitful discussions with Dr. Markus Strobl and Dr. Dmitry N. Dirin. Furthermore, the authors acknowledge the support of R. Schütz, the NECTAR beamline engineer for experimental setup, as well as Dipl. Ing. Armin Kriele for on-site chemistry laboratory support.

\section{REFERENCES}

(1) Eijk, C. W. E. v. Inorganic Scintillators in Medical Imaging. Phys. Med. Biol. 2002, 47, R85-R106.

(2) Lehmann, E. H.; Vontobel, P.; Frei, G.; Brönnimann, C. Neutron Imaging-Detector Options and Practical Results. Nucl. Instrum. Methods Phys. Res., Sect. A 2004, 531, 228-237.

(3) Kardjilov, N.; Manke, I.; Woracek, R.; Hilger, A.; Banhart, J. Advances in Neutron Imaging. Mater. Today 2018, 21, 652-672.

(4) Martz, H. E.; Logan, C. M.; Schneberk, D. J.; Shull, P. J. X-Ray Imaging: Fundamentals, Industrial Techniques and Applications; CRC Press: Boca Raton, 2016.

(5) Berger, M.; Hubbell, J.; Seltzer, S.; Chang, J.; Coursey, J.; Sukumar, R.; Zucker, D.; Olsen, K. Xcom: Photon Cross Sections Database, NIST Standard Reference Database 8 (XGAM). NIST, 2010.

(6) Soppera, N.; Bossant, M.; Dupont, E. JANIS 4: An Improved Version of the NEA Java-Based Nuclear Data Information System. Nucl. Data Sheets 2014, 120, 294-296.

(7) Harvey, J. A.; Hill, N. W. Scintillation Detectors for Neutron Physics Research. Nucl. Instrum. Methods 1979, 162, 507-529.

(8) Vontobel, P.; Lehmann, E. H.; Hassanein, R.; Frei, G. Neutron Tomography: Method and Applications. Phys. B 2006, 385-386, 475-480.

(9) Kardjilov, N.; Manke, I.; Hilger, A.; Strobl, M.; Banhart, J. Neutron Imaging in Materials Science. Mater. Today 2011, 14, $248-$ 256.

(10) Butler, L. G.; Schillinger, B.; Ham, K.; Dobbins, T. A.; Liu, P.; Vajo, J. J. Neutron Imaging of a Commercial Li-Ion Battery during Discharge: Application of Monochromatic Imaging and Polychro- 
matic Dynamic Tomography. Nucl. Instrum. Methods Phys. Res., Sect. A 2011, 651, 320-328.

(11) JEF Report 14: Table of Simple Integral Neutron Cross Section Data from JEF-2.2, ENDF/B-VI, JENDL-3.2, BROND-2 and CENDL2. Nuclear Energy Agency, http://www.oecd-nea.org/dbdata/nds jefreports/, 1994 (accessed 2020-06-03).

(12) Zboray, R.; Adams, R.; Morgano, M.; Kis, Z. Qualification and Development of Fast Neutron Imaging Scintillator Screens. Nucl. Instrum. Methods Phys. Res., Sect. A 2019, 930, 142-150.

(13) Eberhardt, J. E.; Rainey, S.; Stevens, R. J.; Sowerby, B. D.; Tickner, J. R. Fast Neutron Radiography Scanner for the Detection of Contraband in Air Cargo Containers. Appl. Radiat. Isot. 2005, 63, 179-188.

(14) Hausladen, P. A.; Bingham, P. R.; Neal, J. S.; Mullens, J. A.; Mihalczo, J. T. Portable Fast-Neutron Radiography with the Nuclear Materials Identification System for Fissile Material Transfers. Nucl. Instrum. Methods Phys. Res., Sect. B 2007, 261, 387-390.

(15) Buffler, A.; Tickner, J. Detecting Contraband Using Neutrons: Challenges and Future Directions. Radiat. Meas. 2010, 45, 11861192.

(16) Schillinger, B.; Beaudet, A.; Fedrigo, A.; Grazzi, F.; Kullmer, O.; Laaß, M.; Makowska, M.; Werneburg, I.; Zanolli, C. Neutron Imaging in Cultural Heritage Research at the FRM II Reactor of the Heinz Maier-Leibnitz Center. Journal of Imaging 2018, 4, 22.

(17) Day, D. H.; Sinclair, R. N. Neutron Moderator Assemblies for Pulsed Thermal Neutron Time-of-Flight Experiments. Nucl. Instrum. Methods 1969, 72, 237-253.

(18) Milbrath, B. D.; Peurrung, A. J.; Bliss, M.; Weber, W. J. Radiation Detector Materials: An Overview. J. Mater. Res. 2008, 23, 2561-2581.

(19) Berger, M. J.; Coursey, J. S.; Zucker, M. A.; Chang, J., StoppingPower \& Range Tables for Electrons, Protons, and Helium Ions: NIST Standard Reference Database 124; NIST, 2017.

(20) Bravar, U.; Bruillard, P. J.; Flckiger, E. O.; Macri, J. R.; McConnell, M. L.; Moser, M. R.; Ryan, J. M.; Woolf, R. S. Design and Testing of a Position-Sensitive Plastic Scintillator Detector for Fast Neutron Imaging. IEEE Trans. Nucl. Sci. 2006, 53, 3894-3903.

(21) Geppert-Kleinrath, V.; Freeman, M. S.; Hurlbut, C. R.; Merrill, F.; Tinsley, J. R.; Volegov, P.; Wilde, C. A Liquid VI Scintillator Cell for Fast-Gated Neutron Imaging. Rev. Sci. Instrum. 2018, 89, 101142. (22) Mor, I.; Vartsky, D.; Brandis, M.; Goldberg, M. B.; Bar, D.; Mardor, I.; Dangendorf, V.; Bromberger, B. Fast-Neutron Imaging Spectrometer Based on Liquid Scintillator Loaded Capillaries. J. Instrum. 2012, 7, C04021-C04021.

(23) Pozzi, S. A.; Shin, T. H.; Hua, M.; Steinberger, W.; Bourne, M. M.; Clarke, S. D. Recent Advances in Neutron Detection with Organic Scintillators. In Hard X-Ray, Gamma-Ray, and Neutron Detector Physics XXI; San Diego, California, United States, 12 August 2019; edited by James, R. B., Burger, A., Payne, S. A. Proc. SPIE Vol. 11114; SPIE: Bellingham, WA, United States, 2019; 1111409.

(24) Chuirazzi, W. C.; Craft, A. E.; Schillinger, B.; Cool, S.; Oksuz, I. Parametric Evaluation of New Fast Neutron Scintillator Designs; Idaho National Lab.(INL): Idaho Falls, ID, 2019.

(25) Yang, W.; Bin, T.; Heyong, H.; Bin, L.; Ke, T.; Yong, S.; Wei, Y.; Chao, C. The Study of Zinc Sulphide Scintillator for Fast Neutron Radiography. Phys. Procedia 2013, 43, 205-215.

(26) Zboray, R.; Adams, R.; Kis, Z. Scintillator Screen Development for Fast Neutron Radiography and Tomography and Its Application at the Beamline of the $10 \mathrm{MW}$ BNC Research Reactor. Appl. Radiat. Isot. 2018, 140, 215-223.

(27) Guo, J.; Bücherl, T.; Zou, Y.; Guo, Z.; Tang, G. Comparison of the Performance of Different Converters for Neutron Radiography and Tomography Using Fission Neutrons. Nucl. Instrum. Methods Phys. Res., Sect. A 2009, 605, 69-72.

(28) Makowska, M. G.; Walfort, B.; Zeller, A.; Grünzweig, C.; Bücherl, T. Performance of the Commercial PP/ZnS: $\mathrm{Cu}$ and PP/ $\mathrm{ZnS}$ : Ag Scintillation Screens for Fast Neutron Imaging. Journal of Imaging 2017, 3, 60.
(29) Eychmüller, A. Structure and Photophysics of Semiconductor Nanocrystals. J. Phys. Chem. B 2000, 104, 6514-6528.

(30) Kovalenko, M. V.; Manna, L.; Cabot, A.; Hens, Z.; Talapin, D. V.; Kagan, C. R.; Klimov, V. I.; Rogach, A. L.; Reiss, P.; Milliron, D. J.; Guyot-Sionnnest, P.; Konstantatos, G.; Parak, W. J.; Hyeon, T.; Korgel, B. A.; Murray, C. B.; Heiss, W. Prospects of Nanoscience with Nanocrystals. ACS Nano 2015, 9, 1012-1057.

(31) Murray, C. B.; Norris, D. J.; Bawendi, M. G. Synthesis and Characterization of Nearly Monodisperse CdE (E = Sulfur, Selenium, Tellurium) Semiconductor Nanocrystallites. J. Am. Chem. Soc. 1993, 115, 8706-8715.

(32) Kovalenko, M. V.; Protesescu, L.; Bodnarchuk, M. I. Properties and Potential Optoelectronic Applications of Lead Halide Perovskite Nanocrystals. Science 2017, 358, 745.

(33) Robel, I.; Gresback, R.; Kortshagen, U.; Schaller, R. D.; Klimov, V. I. Universal Size-Dependent Trend in Auger Recombination in Direct-Gap and Indirect-Gap Semiconductor Nanocrystals. Phys. Rev. Lett. 2009, 102, 177404.

(34) Brennan, M. C.; Herr, J. E.; Nguyen-Beck, T. S.; Zinna, J.; Draguta, S.; Rouvimov, S.; Parkhill, J.; Kuno, M. Origin of the SizeDependent Stokes Shift in CsPbBr ${ }_{3}$ Perovskite Nanocrystals. J. Am. Chem. Soc. 2017, 139, 12201-12208.

(35) Manna, L.; Scher, E. C.; Alivisatos, A. P. Synthesis of Soluble and Processable Rod-, Arrow-, Teardrop-, and Tetrapod-Shaped CdSe Nanocrystals. J. Am. Chem. Soc. 2000, 122, 12700-12706.

(36) Kunneman, L. T.; Tessier, M. D.; Heuclin, H.; Dubertret, B.; Aulin, Y. V.; Grozema, F. C.; Schins, J. M.; Siebbeles, L. D. A. Bimolecular Auger Recombination of Electron-Hole Pairs in TwoDimensional CdSe and $\mathrm{CdSe} / \mathrm{CdZnS}$ Core/Shell Nanoplatelets. J. Phys. Chem. Lett. 2013, 4, 3574-3578.

(37) Kormilina, T. K.; Cherevkov, S. A.; Fedorov, A. V.; Baranov, A. V. Cadmium Chalcogenide Nano-Heteroplatelets: Creating Advanced Nanostructured Materials by Shell Growth, Substitution, and Attachment. Small 2017, 13, 1702300.

(38) Weidman, M. C.; Goodman, A. J.; Tisdale, W. A. Colloidal Halide Perovskite Nanoplatelets: An Exciting New Class of Semiconductor Nanomaterials. Chem. Mater. 2017, 29, 5019.

(39) Reiss, P.; Protière, M.; Li, L. Core/Shell Semiconductor Nanocrystals. Small 2009, 5, 154-168.

(40) Coropceanu, I.; Bawendi, M. G. Core/Shell Quantum Dot Based Luminescent Solar Concentrators with Reduced Reabsorption and Enhanced Efficiency. Nano Lett. 2014, 14, 4097-4101.

(41) Turtos, R. M.; Gundacker, S.; Omelkov, S.; Auffray, E.; Lecoq, P. Light Yield of Scintillating Nanocrystals under X-Ray and Electron Excitation. J. Lumin. 2019, 215, 116613.

(42) Klassen, N. V.; Kedrov, V. V.; Kurlov, V. N.; Ossipyan, Y. A.; Shmurak, S. Z.; Shmyt'ko, I. M.; Strukova, G. K.; Kobelev, N. P.; Kudrenko, E. A.; Krivko, O. A.; Kiselev, A. P.; Bazhenov, A. V.; Fursova, T. N. Advantages and Problems of Nanocrystalline Scintillators. IEEE Trans. Nucl. Sci. 2008, 55, 1536-1541.

(43) Yukihara, E.; Jacobsohn, L. G.; Blair, M. W.; Bennett, B. L.; Tornga, S. C.; Muenchausen, R. E. Luminescence Properties of CeDoped Oxyorthosilicate Nanophosphors and Single Crystals. J. Lumin. 2010, 130, 2309-2316.

(44) Dujardin, C.; Auffray, E.; Bourret-Courchesne, E.; Dorenbos, P.; Lecoq, P.; Nikl, M.; Vasil'ev, A. N.; Yoshikawa, A.; Zhu, R. Needs, Trends, and Advances in Inorganic Scintillators. IEEE Trans. Nucl. Sci. 2018, 65, 1977-1997.

(45) Padilha, L. A.; Bae, W. K.; Klimov, V. I.; Pietryga, J. M.; Schaller, R. D. Response of Semiconductor Nanocrystals to Extremely Energetic Excitation. Nano Lett. 2013, 13, 925-932.

(46) Williams, R. T.; Wolszczak, W. W.; Yan, X.; Carroll, D. L. Perovskite Quantum-Dot-in-Host for Detection of Ionizing Radiation. ACS Nano 2020, 14, 5161.

(47) Chen, Q.; Wu, J.; Ou, X.; Huang, B.; Almutlaq, J.; Zhumekenov, A. A.; Guan, X.; Han, S.; Liang, L.; Yi, Z.; Li, J.; Xie, X.; Wang, Y.; Li, Y.; Fan, D.; Teh, D. B. L.; All, A. H.; Mohammed, O. F.; Bakr, O. M.; Wu, T.; et al. All-Inorganic Perovskite Nanocrystal Scintillators. Nature 2018, 561, 88-93. 
(48) Zhang, Y.; Sun, R.; Ou, X.; Fu, K.; Chen, Q.; Ding, Y.; Xu, L.-J.; Liu, L.; Han, Y.; Malko, A. V.; Liu, X.; Yang, H.; Bakr, O. M.; Liu, H.; Mohammed, O. F. Metal Halide Perovskite Nanosheet for X-Ray High-Resolution Scintillation Imaging Screens. ACS Nano 2019, 13, $2520-2525$.

(49) Heo, J. H.; Shin, D. H.; Park, J. K.; Kim, D. H.; Lee, S. J.; Im, S. H. High-Performance Next-Generation Perovskite Nanocrystal Scintillator for Nondestructive X-Ray Imaging. Adv. Mater. 2018, 30, 1801743.

(50) He, Y.; Matei, L.; Jung, H. J.; McCall, K. M.; Chen, M.; Stoumpos, C. C.; Liu, Z.; Peters, J. A.; Chung, D. Y.; Wessels, B. W.; Wasielewski, M. R.; Dravid, V. P.; Burger, A.; Kanatzidis, M. G. High Spectral Resolution of Gamma-Rays at Room Temperature by Perovskite CsPbBr 3 Single Crystals. Nat. Commun. 2018, 9, 1609.

(51) Turtos, R. M.; Gundacker, S.; Polovitsyn, A.; Christodoulou, S.; Salomoni, M.; Auffray, E.; Moreels, I.; Lecoq, P.; Grim, J. Q. Ultrafast Emission from Colloidal Nanocrystals under Pulsed X-Ray Excitation. J. Instrum. 2016, 11, P10015-P10015.

(52) Deng, Y.; Lin, X.; Fang, W.; Di, D.; Wang, L.; Friend, R. H.; Peng, X.; Jin, Y. Deciphering Exciton-Generation Processes in Quantum-Dot Electroluminescence. Nat. Commun. 2020, 11, 2309.

(53) Bücherl, T.; Söllradl, S. NECTAR: Radiography and Tomography Station Using Fission Neutrons. Journal of Large-Scale Research Facilities 2015, 1, A19.

(54) Andor's Ikon-L High Dynamic Range CCD Camera. https:// andor.oxinst.com/products/ikon-xl-and-ikon-large-ccd-series/ikon-l936 (accessed 2020-06-30).

(55) Li, C.; Chen, W.; Wu, D.; Quan, D.; Zhou, Z.; Hao, J.; Qin, J.; Li, Y.; He, Z.; Wang, K. Large Stokes Shift and High Efficiency Luminescent Solar Concentrator Incorporated with $\mathrm{CuInS}_{2} / \mathrm{ZnS}$ Quantum Dots. Sci. Rep. 2016, 5, 17777.

(56) McDaniel, H.; Koposov, A. Y.; Draguta, S.; Makarov, N. S.; Pietryga, J. M.; Klimov, V. I. Simple yet Versatile Synthesis of

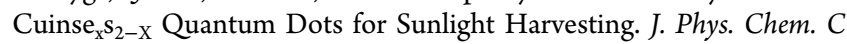
2014, 118, 16987-16994.

(57) Parobek, D.; Roman, B. J.; Dong, Y.; Jin, H.; Lee, E.; Sheldon, M.; Son, D. H. Exciton-to-Dopant Energy Transfer in Mn-Doped Cesium Lead Halide Perovskite Nanocrystals. Nano Lett. 2016, 16, 7376-7380.

(58) Pazos-Outón, L. M.; Szumilo, M.; Lamboll, R.; Richter, J. M.; Crespo-Quesada, M.; Abdi-Jalebi, M.; Beeson, H. J.; Vrućinić, M.; Alsari, M.; Snaith, H. J.; Ehrler, B.; Friend, R. H.; Deschler, F. Photon Recycling in Lead Iodide Perovskite Solar Cells. Science 2016, 351, 1430.

(59) Gan, Z.; Chen, W.; Yuan, L.; Cao, G.; Zhou, C.; Huang, S.; Wen, X.; Jia, B. External Stokes Shift of Perovskite Nanocrystals Enlarged by Photon Recycling. Appl. Phys. Lett. 2019, 114, No. 011906.

(60) Jungwirth, M.; Breitkreutz, H.; Wagner, F. M.; Bücherl, T. Determination of the Photon Spectrum in an Intense Fission Neutron Beam. J. Instrum. 2012, 7, C03022-C03022.

(61) Makii, H.; Nishio, K.; Hirose, K.; Orlandi, R.; Léguillon, R.; Ogawa, T.; Soldner, T.; Hambsch, F.-J.; Astier, A.; Pollitt, A.; Petrache, C.; Tsekhanovich, I.; Mathieu, L.; Aïche, M.; Frost, R.; Czajkowski, S.; Guo, S.; Köster, U. Measurement of High-Energy Prompt Gamma-Rays from Neutron Induced Fission of U-235. EPJ Web Conf. 2017, 146, 04036.

(62) Schindelin, J.; Arganda-Carreras, I.; Frise, E.; Kaynig, V.; Longair, M.; Pietzsch, T.; Preibisch, S.; Rueden, C.; Saalfeld, S.; Schmid, B.; Tinevez, J.-Y.; White, D. J.; Hartenstein, V.; Eliceiri, K.; Tomancak, P.; Cardona, A. Fiji: An Open-Source Platform for Biological-Image Analysis. Nat. Methods 2012, 9, 676-682.

(63) Schneider, C. A.; Rasband, W. S.; Eliceiri, K. W. NIH Image to ImageJ: 25 Years of Image Analysis. Nat. Methods 2012, 9, 671-675. (64) Allison, J.; Amako, K.; Apostolakis, J.; Arce, P.; Asai, M.; Aso, T.; Bagli, E.; Bagulya, A.; Banerjee, S.; Barrand, G.; Beck, B. R.; Bogdanov, A. G.; Brandt, D.; Brown, J. M. C.; Burkhardt, H.; Canal, P.; Cano-Ott, D.; Chauvie, S.; Cho, K.; Cirrone, G. A. P.; et al. Recent
Developments in Geant4. Nucl. Instrum. Methods Phys. Res., Sect. A 2016, 835, 186-225. 